\title{
Expression of RAP1B is associated with poor prognosis and promotes an aggressive phenotype in gastric cancer
}

\author{
YA YANG $^{1 *}$, MIN LI $^{1}$, YAN YAN $^{1}$, JIA ZHANG $^{1}$, KAI SUN $^{1}$, JING-KUN QU $^{1}$, \\ JIAN-SHENG WANG $^{1^{*}}$ and XIAO-YI DUAN ${ }^{2 *}$ \\ ${ }^{1}$ The Second Department of Thoracic Surgery, and ${ }^{2}$ Department of Radiology, \\ The First Affiliated Hospital of Xi'an Jiaotong University, Xi'an, Shaanxi 710061, P.R. China
}

Received May 20, 2015; Accepted June 29, 2015

DOI: $10.3892 / o r .2015 .4234$

\begin{abstract}
Metastasis is the major cause of death among gastric cancer (GC) patients, and altered expression of Ras-related protein RAP1B is associated with cancer development. The present study assessed RAP1B expression ex vivo and the effect of hypoxia-induced RAP1B overexpression on the promotion of the metastatic potential of GC cells in vitro. Immunohistochemistry was used to detect the expression of RAP1B and hypoxia-inducible factor- $1 \alpha$ (HIF-1 $\alpha)$ in 178 GC tissue specimens. GC cell lines were used to assess the effects of hypoxia and RAP1B knockdown with RAP1B small interfering RNA (siRNA). Tumor cell viability was detected by the 3-(4,5-dimethylthiazol-2-yl)-2,5-diphenyltetrazolium bromide (MTT) assay, invasion capacity was evaluated by a Transwell assay, and gene expression was determined by qRT-PCR and western blotting. The data showed that expression levels of RAP1B and HIF-1 $\alpha$ proteins were high in the GC tissue specimens, and RAP1B expression was significantly associated with tumor-node-metastasis (TNM) stage and tumor size, while HIF-1 $\alpha$ expression was significantly associated with TNM stage, Borrmann type and tumor differentiation. Moreover, RAP1B expression was associated with HIF- $1 \alpha$ expression $(r=0.547, \mathrm{P}<0.001)$. The expression of RAP1B and HIF-1 $\alpha$ proteins was associated with a shorter overall survival of patients according to the univariate analysis (log-rank test, $\mathrm{P}<0.01$ ), and RAP1B expression and TNM
\end{abstract}

Correspondence to: Professor Xiao-Yi Duan, Department of Radiology, The First Affiliated Hospital of Xi'an Jiaotong University, 277 West Yanta Road, Xi'an, Shaanxi 710061, P.R. China

E-mail: duanxy@mail.xjtu.edu.cn

Professor Jian-Sheng Wang, The Second Department of Thoracic Surgery, The First Affiliated Hospital of Xi'an Jiaotong University, 277 West Yanta Road, Xi'an, Shaanxi 710061, P.R. China

E-mail:wangjsh@mail.xjtu.edu.cn

*Contributed equally

Key words: gastric cancer, RAP1B, HIF-1 $\alpha$, biomarker, survival, tumor cell invasion stage were independent prognostic predictors in patients using a multivariate analysis $(\mathrm{P}<0.001)$. In vitro, hypoxia induced the invasion of GC cells and the expression of RAP1B and HIF-1 $\alpha(\mathrm{P}<0.05)$; whereas knockdown of RAP1B expression using siRNA inhibited the tumor cell invasion capacity even under hypoxic culture conditions $(\mathrm{P}<0.05)$. In conclusion, the protein expression of RAP1B and HIF- $1 \alpha$ contributed to GC malignant behavior and poor prognosis. Future studies will evaluate whether targeting RAP1B expression can be used as a novel strategy to control GC or as a biomarker for prognosis prediction.

\section{Introduction}

Gastric cancer (GC) is a significant worldwide health issue as it is an aggressive disease and has a continuous impact on global health (1). Due to advancements in early detection, optimal treatment and cancer prevention, the global GC incidence has been declining for the last several decades; however, to date, GC remains the fourth most common cancer and is the second leading cause of cancer-related deaths worldwide $(1,2)$. Most GC patients still face a poor prognosis since treatment for advanced or metastatic GC has made little progress, and the median overall survival in this group of patients is still less than 1 year (3). Geographically, $73 \%$ of GC cases are diagnosed in Asia and $~ 50 \%$ of all GC occurs in China $(4,5)$. Thus, further study is urgently needed to identify and evaluate biomarkers for early detection and prognostic prediction as well as to develop novel strategies for the prevention and control of GC. Like most human cancers, GC development involves the activation of oncogenes and the silencing of tumor suppressor genes. Dysregulated genes also contribute to the transformation of normal cells to malignant cells (6).

Towards this end, our research focuses on Ras-related protein Rap1, which is a member of the Ras superfamily of GTPases. The function of these GTPases in cells is to shuttle between inactive GDP- and active GTP-bound states, thus regulating multiple cellular processes including cell adhesion, migration, polarity, differentiation, growth and angiogenesis $(7,8)$. RAP1B is an isoform of Rap1 and possesses different functions in different cells $(9,10)$. For example, RAP1B participates in endothelial cell adhesion, migration, 
proliferation and capillary tube formation (11). RAP1B also functions as a key suppressor of neutrophil migration and lung inflammation (12). Other studies have shown that altered RAP1B expression occurs in several types of human cancer and that RAP1B promotes cancer growth, invasion and metastasis $(7,11,13-18)$. Specifically, RAP1B expression is upregulated in late-stage ovarian tumors and inhibition of RAP1B expression suppressed tumor cell migration/invasion in vitro and also suppressed the abdominal metastasis of an orthotopic xenograft mouse model (11). However, one study showed that knockdown of RAP1B expression led to a significant recovery of the migratory response to lysophosphatidic acid (LPA) in glioma cells (19). Thus, in the present study, we first detected the expression of RAP1B and hypoxia-inducible factor- $1 \alpha$ (HIF-1 $\alpha$ ) in GC tissue specimens to assess the association with the clinicopathological and survival data from the patients and then assessed the role of RAP1B in GC in vitro. As is well known, hypoxia is a common feature in the tumor microenvironment that impacts tumor cell survival and malignant behaviors (20-22). In addition, the hypoxic microenvironment of a tumor is an independent predictor of a poor prognosis in various types of cancer including GC (23), and the transcription factor HIF-1 $\alpha$ mediates the effects of hypoxia (24). Thus, the present study provides useful information for the future control of GC.

\section{Materials and methods}

Materials. Diaminobenzidine (DAB) was obtained from Golden Bridge International (Beijing, China) and Dulbecco's modified Eagle's medium (DMEM) was obtained from Life Technologies (New York, NY, USA). Fetal bovine serum (FBS), Lipofectamine 2000, TRIzol reagent and 3-(4,5-dimethylthiazol-2-yl)-2,5-diphenyltetrazolium bromide (MTT) were provided by Invitrogen (Carlsbad, CA, USA). The SuperScript II reverse transcriptase kit was purchased from Takara Bio Inc. (Otsu, Shiga, Japan) and the enhanced chemiluminescence (ECL) reagent and polyvinylidene fluoride (PVDF) membranes were from Merck Millipore (Darmstadt, Germany). The RIPA lysis buffer kit was from Santa Cruz Biotechnology (Santa Cruz, CA, USA) and the protein assay kit was purchased from Bio-Rad (Hercules, CA, USA). Cobalt dichloride $\left(\mathrm{CoCl}_{2}\right)$ and other reagents were from Sigma (St. Louis, MO, USA) unless otherwise stated.

Tissue specimens. Tissue specimens from $178 \mathrm{GC}$ patients were collected from consecutive surgical cases at the Department of Surgical Oncology, The First Affiliated Hospital, Medical School, Xi'an Jiaotong University and the Department of Surgical Oncology, The 215th Hospital of Shaanxi Province, between 2004 and 2009. The patients included 125 males and 53 females, ranging between the ages of 25 and 81 years. Histological diagnosis of GC was assessed according to the criteria of staging of the primary tumor/regional lymph nodes/ distant metastasis (TNM) as described in the AJCC Cancer Staging Manual (25). The clinicopathological data of the patients are documented in Table II. In brief, tumors penetrating into the serosa or those involved in adjacent structures were observed in 77 patients $(43.25 \%), 129$ patients $(72.47 \%)$ had lymph node metastases and 40 patients $(22.47 \%)$ had distant metastasis. Twenty-six patients underwent total gastrectomy, whereas the remaining 152 patients underwent partial resection of tumor lesions. Among them, 178 patients did not receive any neoadjuvant or adjuvant chemotherapy before surgery, and 108 of 178 patients received fluorouracil-based postoperative adjuvant chemotherapy. The present study was approved by the Protection of Human Subjects Committee of The First Affiliated Hospital, Medical School, Xi'an Jiaotong University and complies with the Helsinki Declaration.

Immunohistochemistry. Tissue specimens were fixed in a buffered formalin solution and embedded in paraffin for serial sections (4- $\mu \mathrm{m}$ in thickness) that were mounted onto charged glass slides. For immunohistochemistry, the tissue sections were subjected to deparaffinization and rehydration and then to antigen retrieval in a citrate buffer at $\mathrm{pH} 6.0$ in a microwave. The sections were then subsequently incubated with a goat polyclonal antibody against RAP1B (sc-1481; Santa Cruz Biotechnology) at a dilution of 1:600 or anti-HIF-1 $\alpha$ (bs0737R; Biosynthesis Biotechnology, Beijing, China) at a dilution of 1:500 overnight at $4^{\circ} \mathrm{C}$. The streptavidin-peroxidase technique (SP-9001 and PV-9003; Golden Bridge International) was used to amplify the signal, and the sections were incubated with $0.02 \%$ DAB solution to develop the positive color reaction, followed by brief counterstaining with hematoxylin. Some duplicated sections were also incubated with an irrelevant rabbit antiserum to replace the first antibody which served as a negative control.

Evaluation of immunostained sections. The immunostained tissue sections were reviewed and scored under a microscope independently by two experienced pathologists, who were blinded to the clinical data with consensus. The staining results for RAP1B and HIF-1 $\alpha$ proteins were scored semiquantitatively by addition of the immunostaining intensity and the percentage of positive tumor cells. The percentage of positive malignant cells was determined in at least five fields under a 4400 magnification and was averaged as follows: no staining or $<5 \%, 0$ points; between 5 and 25\%, 1 point; between 26 and $50 \%, 2$ points; between 51 and $75 \%, 3$ points; and $>75 \%$, 4 points. The staining intensity was scored as follows: no coloring, 0 points; slightly yellow, 1 point; brown yellow, 2 points; and tan stains, 3 points. If the sum of these two scores was $\leq 5$, the section was defined as having low expression; whereas $\geq 6$ was defined as high expression.

Cell lines and culture. The human GC cell lines MKN28, BGC823 and SGC7901 were obtained from the Cell Bank of Shanghai (Shanghai, China) and cultured in DMEM with high glucose and supplemented with $10 \%$ heat-inactivated FBS at $37^{\circ} \mathrm{C}$ in a humidified incubator under an atmosphere of $5 \%$ carbon dioxide. All experiments were performed with cells in the logarithmic phase of growth.

MTT assay. Cancerous cells were seeded into 96-well plates at a density of $10^{4}$ cells/well and incubated at $37^{\circ} \mathrm{C}$ for $24 \mathrm{~h}$. Next, fresh growth medium containing the various concentrations of $\mathrm{CoCl}_{2}$ (at concentrations of 50-300 $\mu \mathrm{M}$ ) was added into each well. Following incubation at $37^{\circ} \mathrm{C}$ for $24 \mathrm{~h}$, the cell viability was assessed using the MTT colorimetric assay. All 


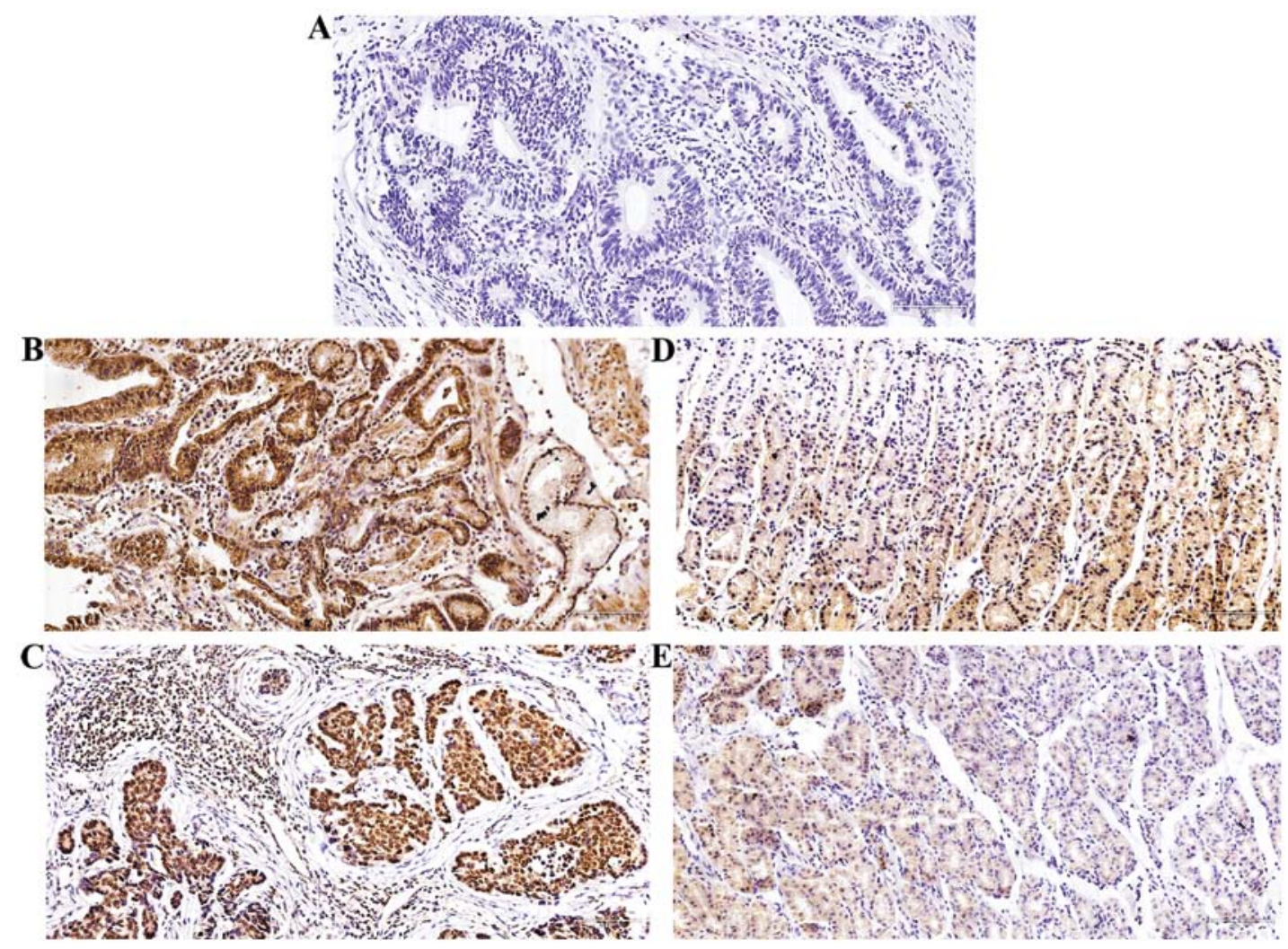

Figure 1. Immunohistochemical analysis of RAP1B and HIF-1 $\alpha$ expression in the gastric cancer (GC) tissue specimens. (A) Negative staining of RAP1B and HIF-1 $\alpha$ proteins in GC tissue specimens. (B) Typical immunohistological features with high RAP1B expression in GC tissue specimens. The RAP1B staining was localized predominantly in the nuclei and cytosol. (C) Typical immunohistological features with high HIF-1 $\alpha$ expression in GC tissue specimens. (D) RAP1B expression in gastric para-cancer tissue specimens. (E) HIF-1 $\alpha$ expression in gastric para-cancer tissue specimens. Magnification, x200.

experiments were conducted in triplicate and repeated at least three times.

Transwell assay. To assess the alteration of tumor cell invasion capacity, a Transwell assay was performed using a membrane invasion culture system (Corning, Corning, NY, USA), following the standard protocol. Briefly, control or siRAP1B-transfected GC cells $\left(3-5 \times 10^{4} /\right.$ well) were resuspended in $200 \mu \mathrm{l}$ of serum-free DMEM with high glucose containing 150 or $200 \mu \mathrm{M} \mathrm{CoCl}_{2}$ and were added into the upper compartment of the Transwell chambers. A total of $600 \mu \mathrm{l}$ of DMEM with high glucose containing $20 \%$ (v/v) FBS and 150 or $200 \mu \mathrm{M} \mathrm{CoCl}_{2}$ was added into the bottom chamber as a source of chemoattractant. After incubation at $37^{\circ} \mathrm{C}$ in a $5 \% \mathrm{CO}_{2}$ incubator for $24 \mathrm{~h}$, the non-invaded tumor cells on the upper surface of the membrane were removed with a cotton swab, and the tumor cells that invaded into the lower surface of the membrane were fixed with $4 \%$ paraformaldehyde, stained with $1 \%$ crystal purple, reviewed and randomly counted in five visual fields of each membrane at a magnification of x 200 under a light microscope. Each experiment was performed in triplicate and repeated once.

Transfection and siRNA. Small interfering RNA (siRNA) molecules targeting human RAP1B were designed and synthesized by GenePharma Co. (Shanghai, China). The target sequences were as follows: siRAP1B-439, 5'-GAG GGA UUU AUA CAU GAA ATT-3' and 5'-UUU CAU GUA UAA AUC
CCU CTT-3'; siRAP1B-501, 5'-CCA CAU UUA ACG AUU UAC ATT-3' and 5'-UGU AAA UCG UUA AAU GUG GT-3'; siRAP1B-722, 5'-GUG CGG CAA AUU AAC AGA ATT-3' and 5'-UUC UGU UAA UUU GCC GCA CTT-3'; and negative control siRNA, 5'-UUC UCC GAA CGU GUC ACG UTT-3' and 5'-ACG UGA CAC GUU CGG AGA ATT-3'. The GC cells were grown and transfected with the negative control or RAP1B siRNAs using Lipofectamine 2000 according to the manufacturer's protocol. After transfection for $48 \mathrm{~h}$, the cells were harvested for RNA isolation and western blot analysis of RAP1B expression.

RNA isolation and quantitative PCR. Total cellular RNA was isolated using a TRIzol reagent and reversely transcribed into cDNA using the SuperScript II reverse transcriptase kit, according to the manufacturer's instructions. The synthesized cDNA was then subjected to qPCR amplification using primers: [RAP1B, 5'-TTT ATT CCA TCA CAG CAC AGT CC-3' and 5'-TTT CTG TTA ATT TGC CGC ACT AGG-3'; glyceraldehydes-3-phosphate dehydrogenase (GAPDH), 5'-CTG GGG CTC ATT TG-3' and 5'-CAT CCA CAG TCT TC-3'] under the following conditions: $95^{\circ} \mathrm{C}$ for $30 \mathrm{sec}$ and 30 cycles of $95^{\circ} \mathrm{C}$ for $5 \mathrm{sec}, 60^{\circ} \mathrm{C}$ for $30 \mathrm{sec}$ and $72^{\circ} \mathrm{C}$ for $60 \mathrm{sec}$ for 30 cycles. The quantity of each transcript was normalized to that of GAPDH.

Protein extraction and western blotting. Whole-cell lysates were prepared from cell lines using the RIPA lysis buffer kit, and the protein concentrations were quantified using the 
Table I. Differential expression of RAP1B and HIF-1 $\alpha$ proteins in gastric cancer (GC) tissues vs. para-cancer normal mucosae.

\begin{tabular}{|c|c|c|c|c|c|c|}
\hline \multirow[b]{2}{*}{ Group } & \multicolumn{2}{|c|}{ RAP1B } & \multirow[b]{2}{*}{ P-value } & \multicolumn{2}{|c|}{ HIF- $1 \alpha$} & \multirow[b]{2}{*}{ P-value } \\
\hline & + & - & & + & - & \\
\hline Cancer & 121 & 57 & & 129 & 49 & \\
\hline Para-carcinoma & 40 & 138 & $<0.001$ & 60 & 118 & $<0.001$ \\
\hline Total & 161 & 195 & & 189 & 167 & \\
\hline
\end{tabular}

HIF-1 $\alpha$, hypoxia-inducible factor- $1 \alpha$; GC, gastric cancer.

Bio-Rad protein assay. Equal amounts of protein samples were separated onto a 10 or $12 \%$ sodium dodecyl sulfate-polyacrylamide gel using sodium dodecyl sulfate-polyacrylamide gel electrophoresis (SDS-PAGE) and were then transferred onto a PVDF membrane. For western blotting, the membranes were probed with antibodies against RAP1B and HIF-1 $\alpha$. Specifically, the membranes were blocked in tris-buffered saline with Tween-20 containing 5\% non-fat dry milk and were then incubated overnight with a primary antibody, followed by horseradish peroxidase-conjugated secondary antibodies at room temperature. The blots were visualized with the ECL detection system. $\beta$-actin was used as an internal control and the blots were analyzed densitometrically using Quantity One software (Bio-Rad).

Statistical analysis. Statistical analysis was performed using the SPSS software package version 16.0 (SPSS, Inc., Chicago, IL, USA). The Chi-square test was used to analyze the association of RAP1B and HIF-1 $\alpha$ expression with the clinicopathological variables. The Spearman's rank correlation coefficient test was performed to associate HIF-1 $\alpha$ expression with RAP1B expression in tissue specimens. Overall survival was defined as the time from the date of surgery to the date of last follow-up or death of the patients. Survival curves were calculated using the Kaplan-Meier method and compared using the log-rank test. Multivariate analysis using Cox's proportional hazard model was used to assess prognostic factors. For each treatment and control, data from the independent replicate trials were pooled, and the results were expressed as the means \pm standard error. All continuous data were tested for normality with the Kolmogorov-Smirnov test. The Student's t-test or one-way analysis of variance was used to compare normally distributed variables. A P-value of $\leq 0.05$ was considered to indicate a statistically significant result.

\section{Results}

Differential expression of RAPIB and HIF-1 $\alpha$ proteins is associated with the clinicopathological data of the GC patients. In the present study, we first assessed the protein expression of RAP1B and HIF- $1 \alpha$ using immunohistochemistry in 178 GC tissue specimens (Fig. 1A as a negative control) and found that RAP1B protein was localized in the nuclei or cytosol (Fig. 1B), while HIF-1 $\alpha$ protein was predominantly in the nuclei; concomitant cytoplasmic staining was ignored since HIF-1 $\alpha$ protein is functionally active in the nuclei (Fig. 1C). Expression of these two proteins was significantly different between the cancer and para-cancer normal tissues (Table I). Expression of RAP1B protein was significantly associated with tumor size (diameter), depth of invasion, regional lymph nodes, distant metastasis and TNM staging, yet not with gender, age, tumor differentiation or Borrmann type (Table II). Moreover, HIF-1 $\alpha$ expression was associated with these clinicopathological variables, except for gender, age and tumor diameter. In addition, RAP1B expression was associated with HIF-1 $\alpha$ expression in the GC tissues. Specifically, Spearman's rank correlation coefficient test showed that there were 98 cancer cases expressing a high level of RAP1B vs. 96 cases with HIF-1 $\alpha$ expression (Table III), revealing a positive association $(r=0.547, \mathrm{P}<0.001)$.

Association of RAPIB and HIF-1 $\alpha$ expression with survival of the GC patients. The Kaplan-Meier curves and the log-rank test data showed that patients with a high RAP1Bexpressing tumor had a statistically significant poor overall survival compared to the patients with a low RAP1Bexpressing tumor (Fig. 2A and Table IV); 30 months [95\% confidence interval (CI), 27.01-32.98] vs. 49 months (95\% CI, 41.20-56.79; $\mathrm{P}<0.001)$. The same was true for HIF-1 $\alpha$ expression (Fig. 2B and Table IV). Furthermore, TNM stage and tumor size (diameter) were all associated with a poor overall survival of patients $(\mathrm{P}<0.05)$. The multivariate Cox regression model showed that RAP1B expression $[\mathrm{P}<0.001$, relative risk $(\mathrm{RR})=2.329,95 \% \mathrm{CI}, 1.600-3.390]$ and TNM stage $(\mathrm{P}<0.001$, $\mathrm{RR}=1.708,95 \% \mathrm{CI}, 1.318-2.112$ ) were independent prognostic indicators for survival of GC patients (Table V).

Hypoxia promotes $G C$ cell invasion capacity in vitro. Our ex vivo data demonstrated that RAP1B and HIF- $1 \alpha$ protein expression was associated with malignant behaviors of GC and a poor overall survival; thus, we further assessed the role of hypoxia in the regulation of GC phenotypes and gene expression by treating tumor cells with $\mathrm{CoCl}_{2}$ to mimic in vivo hypoxic conditions. The cell viability MTT assay assessed the proper concentrations of $\mathrm{CoCl}_{2}$ in vitro (Fig. 3). Next, we utilized the Transwell tumor cell invasion assay to detect the invasion capacity of MKN28, BGC823 and SGC7901 cells and found that the numbers of invaded tumor cells were increased following treatment with $\mathrm{CoCl}_{2}(\mathrm{P}<0.05$; Fig. 4). These results suggest that hypoxia increases the invasion capacity of GC cells. 
Table II. Association of RAP1B and HIF1 $\alpha$ expression with clinicopathological data from the GC patients.

\begin{tabular}{|c|c|c|c|c|c|c|c|}
\hline \multirow[b]{2}{*}{ Variable } & \multirow[b]{2}{*}{$\mathrm{N}$} & \multicolumn{2}{|c|}{ RAP1B } & \multirow[b]{2}{*}{ P-value } & \multicolumn{2}{|c|}{ HIF-1 $\alpha$} & \multirow[b]{2}{*}{ P-value } \\
\hline & & High & Low & & High & Low & \\
\hline \multicolumn{8}{|l|}{ Gender } \\
\hline Male & 125 & 71 & 54 & & 72 & 53 & \\
\hline Female & 53 & 27 & 26 & 0.473 & 24 & 29 & 0.132 \\
\hline \multicolumn{8}{|l|}{ Age (years) } \\
\hline$\leq 60$ & 94 & 48 & 46 & & 50 & 44 & \\
\hline$>60$ & 84 & 50 & 34 & 0.2577 & 46 & 38 & 0.834 \\
\hline \multicolumn{8}{|l|}{ TNM stage } \\
\hline I & 21 & 6 & 15 & & 9 & 12 & \\
\hline II & 34 & 17 & 17 & & 11 & 23 & \\
\hline III & 83 & 45 & 38 & & 46 & 37 & \\
\hline IV & 40 & 30 & 10 & 0.005 & 30 & 10 & 0.002 \\
\hline \multicolumn{8}{|c|}{ Depth of invasion } \\
\hline $\mathrm{T} 1$ & 16 & 2 & 14 & & 8 & 8 & \\
\hline $\mathrm{T} 2$ & 18 & 11 & 7 & & 4 & 14 & \\
\hline T3 & 67 & 40 & 27 & & 40 & 27 & \\
\hline $\mathrm{T} 4$ & 77 & 45 & 32 & 0.005 & 44 & 33 & 0.035 \\
\hline \multicolumn{8}{|c|}{ Lymph node metastasis } \\
\hline No & 49 & 21 & 28 & & 21 & 28 & \\
\hline N1 & 36 & 17 & 19 & & 15 & 21 & \\
\hline $\mathrm{N} 2$ & 35 & 17 & 18 & & 19 & 16 & \\
\hline N3 & 58 & 43 & 15 & 0.005 & 41 & 17 & 0.011 \\
\hline \multicolumn{8}{|c|}{ Distant metastasis } \\
\hline M0 & 138 & 68 & 70 & & 66 & 72 & \\
\hline M1 & 40 & 30 & 10 & 0.004 & 30 & 10 & 0.002 \\
\hline \multicolumn{8}{|l|}{ Borrmann } \\
\hline I & 22 & 10 & 12 & & 6 & 16 & \\
\hline II & 55 & 27 & 28 & & 30 & 25 & \\
\hline III & 62 & 38 & 24 & & 35 & 27 & \\
\hline IV & 39 & 23 & 16 & 0.419 & 25 & 14 & 0.019 \\
\hline \multicolumn{8}{|c|}{ Differentiation } \\
\hline High & 12 & 4 & 8 & & 5 & 7 & \\
\hline Moderate & 57 & 30 & 27 & & 24 & 33 & \\
\hline Poor & 109 & 64 & 45 & 0.222 & 67 & 42 & 0.040 \\
\hline \multicolumn{8}{|c|}{ Tumor diameter $(\mathrm{cm})$} \\
\hline$<3$ & 44 & 17 & 27 & & 18 & 26 & \\
\hline $3-5$ & 54 & 33 & 21 & & 32 & 22 & \\
\hline$>5$ & 80 & 48 & 32 & 0.041 & 46 & 34 & 0.104 \\
\hline
\end{tabular}

Hypoxia induces RAPIB and HIF-1 $\alpha$ expression in GC cells. In order to verify whether hypoxia regulates RAP1B expression and induces GC cell invasion, we treated MKN28, BGC823 and SGC7901 cells with 150 or $200 \mu \mathrm{M} \mathrm{CoCl}{ }_{2}$ for $24 \mathrm{~h}$ and found that the expression of both RAP1B mRNA and protein was upregulated ( $\mathrm{P}<0.05$; Fig. 5); similarly, HIF- $1 \alpha$ was upregulated at the protein level $(\mathrm{P}<0.05$; Fig. 5B). Thus, hypoxia induced RAP1B and HIF-1 $\alpha$ expression in GC cells.
Knockdown of RAP1B expression suppresses tumor cell invasion capacity under hypoxic conditions. To illuminate RAP1B function in GC cells, we knocked down RAP1B using siRNAs and found that siRAP1B-501 was shown to have the best efficiency; thus, it was used for the next experiments (Fig. 3D). We then transfected negative control siRNA and siRAP1B501 into GC cells, treated the cells with 150 or $200 \mu \mathrm{M}$ $\mathrm{CoCl}_{2}$ for $24 \mathrm{~h}$, and performed the Transwell tumor cell 
Table III. Association of HIF1 $\alpha$ expression with RAP1B expression.

\begin{tabular}{llllll}
\hline & \multicolumn{2}{c}{ RAP1B } & & \\
\cline { 2 - 3 } & High & Low & & r & P-value \\
\hline HIF-1 $\alpha$ & & & & \\
High & 77 & 19 & & \\
Low & 21 & 61 & & 0.547 & $<0.001$ \\
\hline
\end{tabular}

A

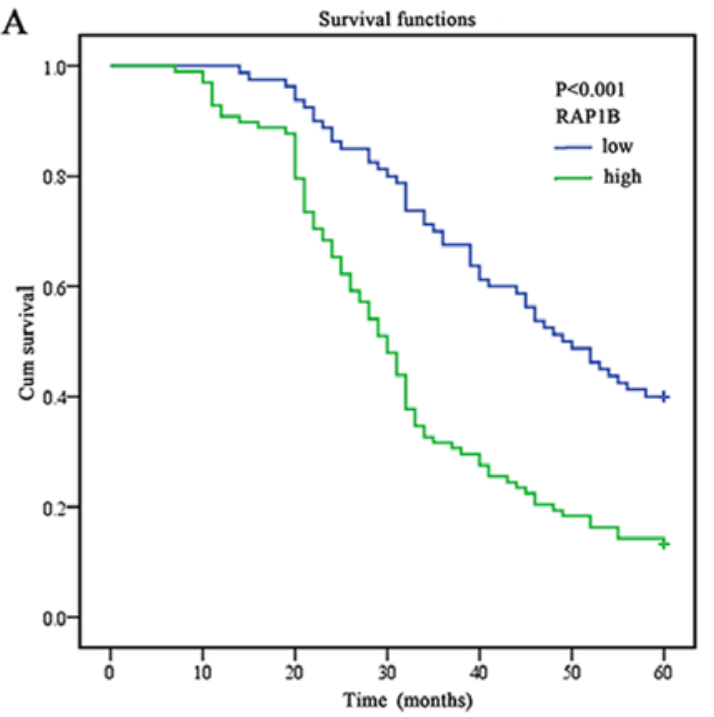

B

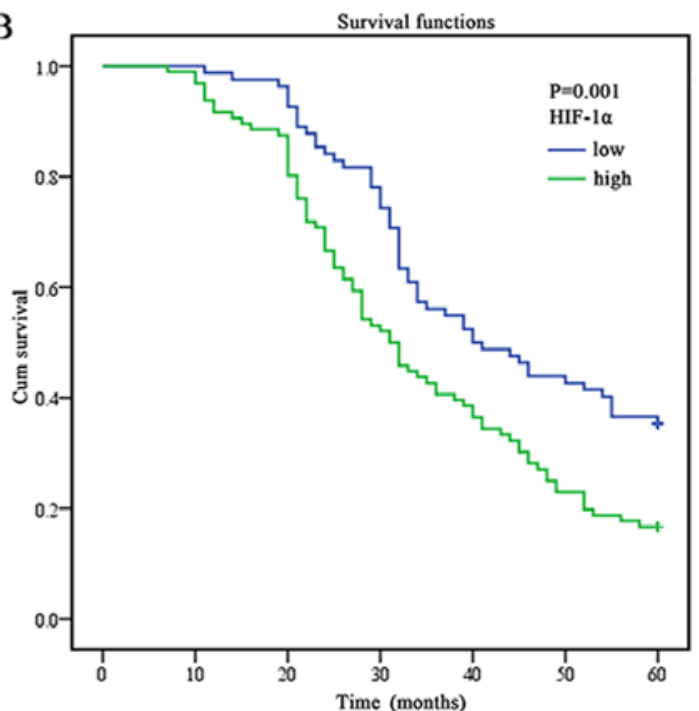

Figure 2. Kaplan-Meier curves of the gastric cancer (GC) patients stratified by RAP1B and HIF-1 $\alpha$ expression. Cumulative overall survival differences between patients with high and low expression of these two proteins. Patients with high protein expression levels showed a poorer overall survival using the log-rank test. (A) RAP1B ( $\mathrm{P}<0.001)$; (B) HIF-1 $\alpha(\mathrm{P}=0.001)$.

invasion assay. Our data showed that MKN28, BGC823 and SGC7901 cells all demonstrated reduced invasion capacity, even after $\mathrm{CoCl}_{2}$ treatment (Fig. 6), compared to the negative
Table IV. Univariate analysis of overall survival.

\begin{tabular}{lrrcr}
\hline & & \multicolumn{2}{c}{ Overall survival } & \\
\cline { 3 - 4 } Variable & $\mathrm{N}$ & Median & $95 \% \mathrm{CI}$ & P-value \\
\hline RAP1B & & & & \\
High & 98 & $30.00 \pm 1.52$ & $27.01-32.98$ & \\
Low & 80 & $49.00 \pm 3.97$ & $41.20-56.79$ & $<0.001$ \\
HIF1 $\alpha$ & & & & \\
High & 96 & $31.00 \pm 2.10$ & $26.88-35.11$ & \\
Low & 82 & $40.00 \pm 4.98$ & $30.23-49.76$ & 0.001 \\
Gender & & & & \\
Male & 125 & $34.00 \pm 2.97$ & $28.16-39.83$ & \\
Female & 53 & $38.00 \pm 3.63$ & $30.86-45.13$ & 0.973 \\
Age (years) & & & & \\
$\leq 60$ & 94 & $40.00 \pm 3.87$ & $32.40-47.59$ & \\
$>60$ & 84 & $32.00 \pm 1.13$ & $29.77-34.22$ & 0.223 \\
TNM & & & & \\
I & 21 & $49.00 \pm 3.21$ & $42.69-66.71$ & \\
II & 34 & $45.00 \pm 5.83$ & $33.57-56.42$ & \\
III & 83 & $34.00 \pm 2.27$ & $29.53-38.46$ & \\
IV & 40 & $29.00 \pm 1.57$ & $25.91-32.08$ & $<0.001$
\end{tabular}

Depth of

invasion

$\begin{array}{llll}\text { T1 } & 16 & 46.00 \pm 8.66 & 29.01-62.98 \\ \text { T2 } & 18 & 52.00 \pm 15.91 & 20.81-83.18 \\ \text { T3 } & 67 & 35.00 \pm 4.60 & 25.98-44.01 \\ \text { T4 } & 77 & 31.00 \pm 1.45 & 28.14-33.86\end{array}$

Lymph node metastasis

$\begin{array}{lllll}\text { N0 } & 49 & 55.00 \pm 9.79 & 35.79-74.20 & \\ \text { N1 } & 36 & 43.00 \pm 7.80 & 27.22-58.78 & \\ \text { N2 } & 35 & 36.00 \pm 3.54 & 29.04-42.95 & \\ \text { N3 } & 58 & 29.00 \pm 2.53 & 24.03-33.96 & <0.001\end{array}$

Distant

metastasis

$\begin{array}{lrrrr}\text { M0 } & 138 & 41.00 \pm 3.41 & 34.31-47.68 & \\ \text { M1 } & 40 & 31.00 \pm 2.37 & 26.35-35.65 & <0.001 \\ \text { Borrmann } & & & & \\ \text { I } & 22 & 39.00 \pm 7.62 & 24.06-53.93 & \\ \text { II } & 55 & 40.00 \pm 3.69 & 32.76-47.23 & \\ \text { III } & 62 & 34.00 \pm 3.50 & 27.14-40.85 & \\ \text { IV } & 39 & 30.00 \pm 1.77 & 27.51-34.48 & 0.502 \\ \text { Differentiation } & & & & \\ \quad \text { High } & 12 & 40.00 \pm 6.06 & 28.11-51.88 & \\ \quad \text { Moderate } & 57 & 39.00 \pm 6.11 & 26.97-51.02 & \\ \quad \text { Poor } & 109 & 32.00 \pm 1.74 & 28.59-35.41 & 0.107\end{array}$

Tumor diameter

(cm)

$\begin{array}{lllll}<3 & 44 & 46.00 \pm 5.20 & 35.79-56.20 & \\ 3-5 & 54 & 34.00 \pm 1.83 & 30.40-37.51 & \\ >5 & 80 & 32.00 \pm 0.98 & 30.06-33.93 & 0.019\end{array}$

CI, confidence interval. 

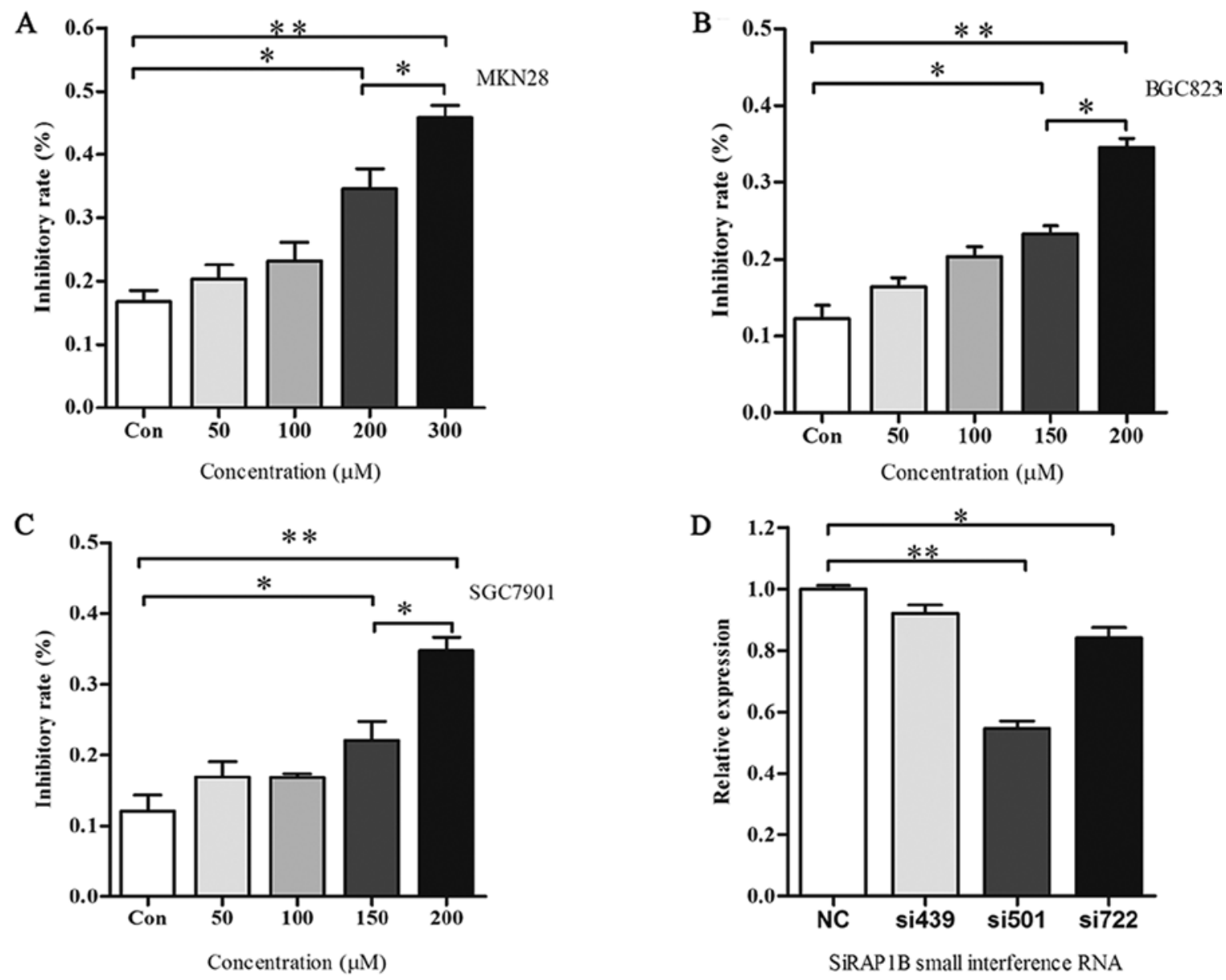

Figure 3. Inhibitory effect of $\mathrm{CoCl}_{2}$ on the viability of gastric cancer (GC) cells. (A) MKN28, (B) BGC823 and (C) SGC7901 cell viability after treatment with different concentrations of $\mathrm{CoCl}_{2}$ by MTT assay. (D) The GC cells were transfected with negative control siRNA or RAP1B siRNAs (siRAP1B-439, siRAP1B-501 and siRAP1B-722) and then subjected to qRT-PCR analysis of RAP1B expression. The results were normalized to GAPDH for the negative control vs. siRNA group (mean \pm standard error) $\left({ }^{*} \mathrm{P}<0.05\right.$ and $\left.{ }^{* *} \mathrm{P}<0.001\right)$.

Table V. Multivariate Cox proportional hazard analysis of overall survival.

\begin{tabular}{lccr}
\hline & \multicolumn{2}{c}{ Overall survival } & \\
\cline { 2 - 3 } Variable & RR & $95 \%$ CI & P-value \\
\hline TNM & 1.708 & $1.318-2.112$ & $<0.001$ \\
RAP1B & 2.329 & $1.600-3.390$ & $<0.001$ \\
Gender & 0.909 & $0.609-1.358$ & 0.643 \\
Age (years) & 1.136 & $0.939-1.844$ & 0.110 \\
HIF1 $\alpha$ & 1.374 & $0.945-1.997$ & 0.096 \\
Borrmann & 0.767 & $0.596-1.034$ & 0.082 \\
Differentiation & 1.213 & $0.974-1.555$ & 0.126 \\
Tumor diameter & 1.357 & $0.960-1.918$ & 0.084 \\
\hline
\end{tabular}

$\mathrm{RR}$, relative risk; TNM, tumor-node-metastasis; CI, confidence interval.

control siRNA-transfected cells after treatment with $\mathrm{CoCl}_{2}$ $(\mathrm{P}<0.001)$. However, knockdown of RAP1B expression did not repress HIF-1 $\alpha$ expression under hypoxic conditions (Fig. 7).
Hence, RAP1B plays an important role in GC invasion under a hypoxic environment.

\section{Discussion}

RAP1B is a member of the Ras superfamily of GTPases (10), and altered RAP1B expression is associated with human carcinogenesis $(7,11,13,14,16,18)$. In the present study, we first analyzed RAP1B and HIF-1 $\alpha$ expression in GC tissue specimens and found that RAP1B was highly expressed in GC tissues and that its expression was associated with malignant behaviors and a poor overall survival of GC patients. RAP1B expression was also highly associated with HIF-1 $\alpha$ expression and the latter was associated with malignant behaviors and a poor overall survival of GC patients, as well. Next, we performed in vitro experiments to assess the role of RAP1B in GC phenotypes and gene expression. Our current data showed that hypoxia induced the expression of RAP1B and HIF-1 $\alpha$ as well as GC cell invasion, whereas knockdown of RAP1B expression suppressed the tumor cell invasion capacity under a hypoxic environment. However, knockdown of RAP1B expression did not alter the HIF- $1 \alpha$ protein levels in the GC cells, which may indicate that RAP1B is a downstream gene of HIF-1 $\alpha$. Our current data demonstrated that the protein expression of RAP1B and HIF-1 $\alpha$ contributed to the malignant 
A

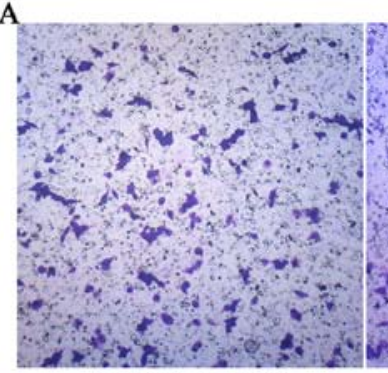

MKN28 $\mathrm{CoCl}_{2}(-)$

$\mathrm{C}$

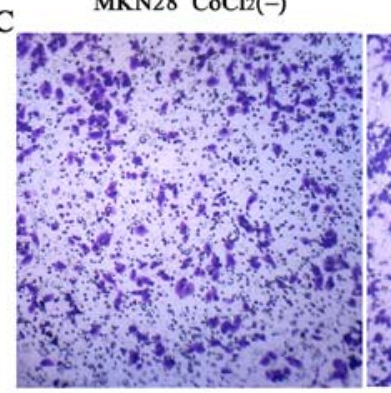

$\mathrm{SGC7901} \mathrm{CoCl}_{2}(-)$

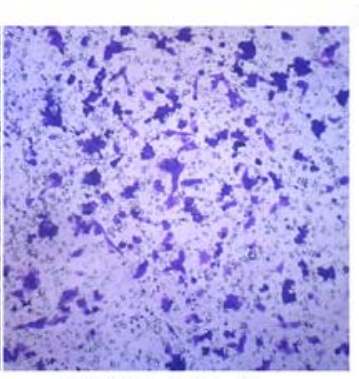

MKN28 $\mathrm{CoCl}_{2}(+)$

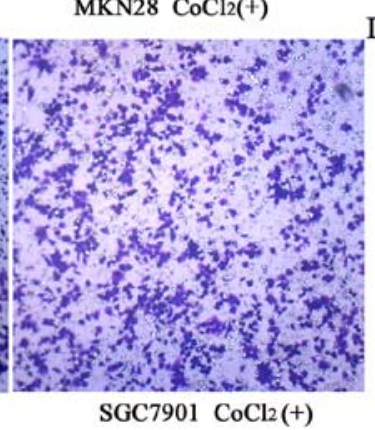

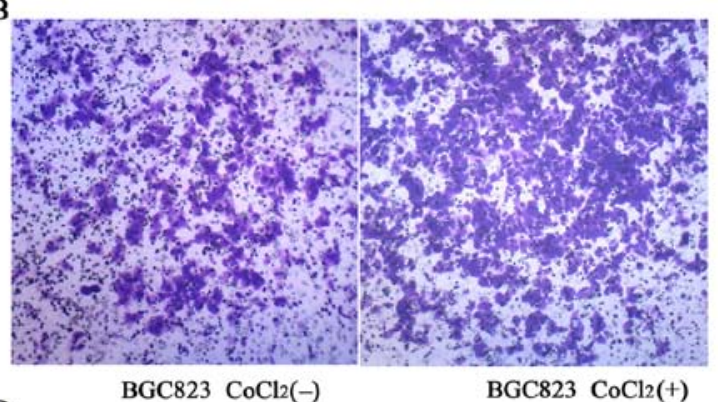

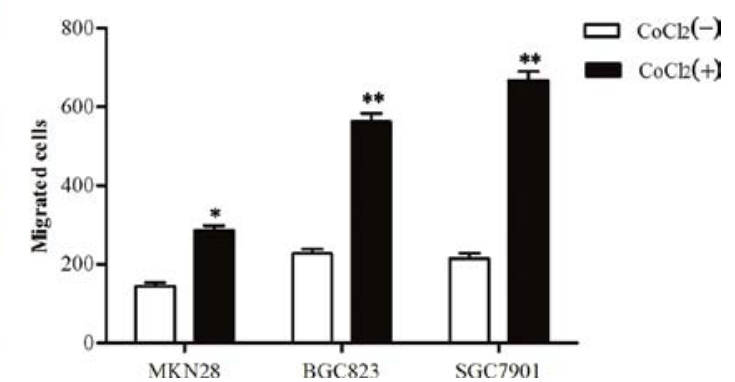

Figure 4. Hypoxia promotes the invasion capacity of gastric cancer (GC) cells. After incubation with $\mathrm{CoCl}_{2}$ for $24 \mathrm{~h}$, images were captured under a phasecontrast microscope. (A) MKN28, (B) BGC823, (C) SGC7901 cells; and (D) quantified data of A-C ( ( $^{*}<0.05$ and $\left.{ }^{* *} \mathrm{P}<0.001\right)$.

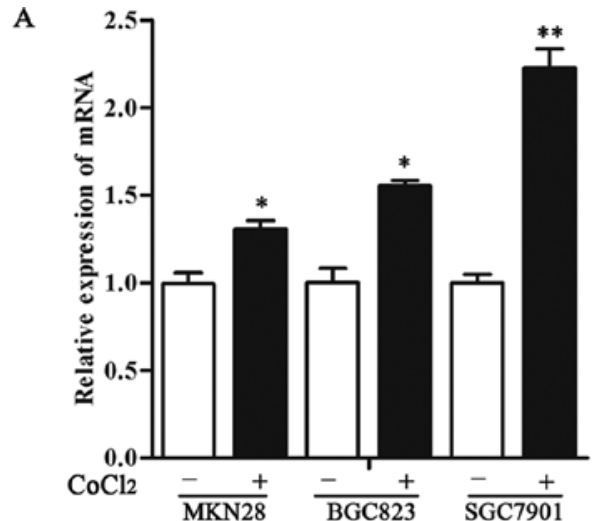

B
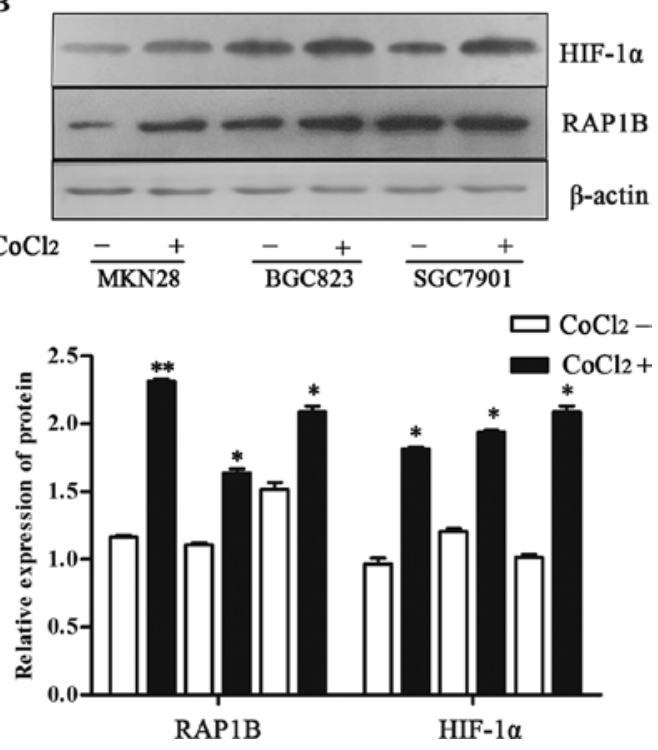

Figure 5. Expression of RAP1B and HIF-1 $\alpha$ in gastric cancer (GC) cells cultured under a hypoxic condition. (A) qRT-PCR. Expression of RAP1B mRNA in GC cells treated with $\mathrm{CoCl}_{2}$ and then subjected to qRT-PCR analysis and (B) western blotting. The duplicate cells were subjected to western blot analysis of RAP1B and HIF-1 $\alpha$ expression. The graph shows the quantified data of the western blot analyses $\left({ }^{*} \mathrm{P}<0.05\right.$ and $\left.{ }^{* *} \mathrm{P}<0.001\right)$. behavior and poor prognosis of GC. Future studies will evaluate whether targeting RAP1B expression can be used as a novel strategy to control GC or as a biomarker for prognosis prediction.

In the present study, we found that RAP1B protein was localized in the nuclei or cytosol, which is consistent with a previous study (26) showing serum-induced nuclear translocation of RAP1B protein in oral cancer cells; whereas under serum-free conditions, RAP1B was localized almost exclusively in the cytoplasm. The phosphorylation of RAP1B protein has been shown to promote the cytosolic and nuclear accumulation of non-prenylated RAP1B (27). Depending on the cell type and conditions, RAP1B expression suppresses or activates cell migration. In the present study, we found that RAP1B expression promoted the invasion capacity of GC cells under hypoxic conditions. In other types of cancer, RAP1B expression was found to be downregulated by miR149 at the transcript level, and RAP1B depletion inhibited the migration and invasion of MDA-MB-231 cells and suppressed the basal level of Rac activity (14). Similarly, low-miR-708 and high-RAP1B were detected in late-state ovarian tumors, and glucocorticoid treatments increased the expression of miR-708, leading to RAP1B inhibition and suppression of ovarian cancer cell migration/invasion and impairment of abdominal metastasis in an orthotopic xenograft mouse model (11). However, another study demonstrated that isoproterenol-induced $\beta 2$-adrenergic receptor stimulation in glioma cells suppressed the migratory response to LPA through the Epac/RAP1Bdependent pathway (19). RAP1B had an inverse function in tumor cell migration; thus, this finding indicates that RAP1B has differential functions in different tumor cells, with various stimulating factors and through different signaling pathways. Although the present study demonstrated that RAP1B plays an important role in GC invasion and metastasis both ex vivo and in vitro, further investigation of RAP1B is warranted. 

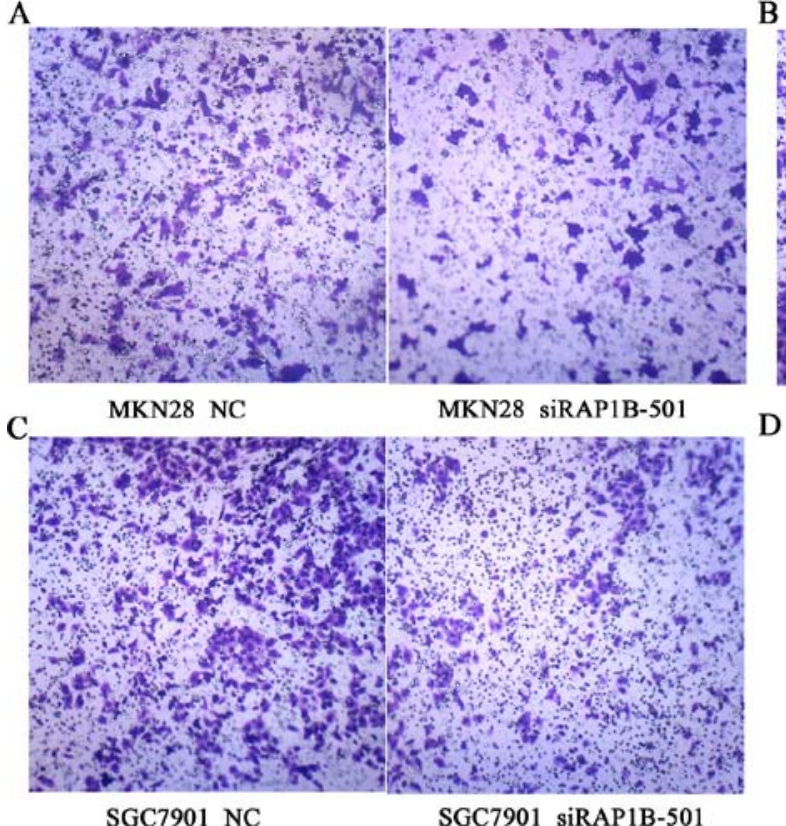

B
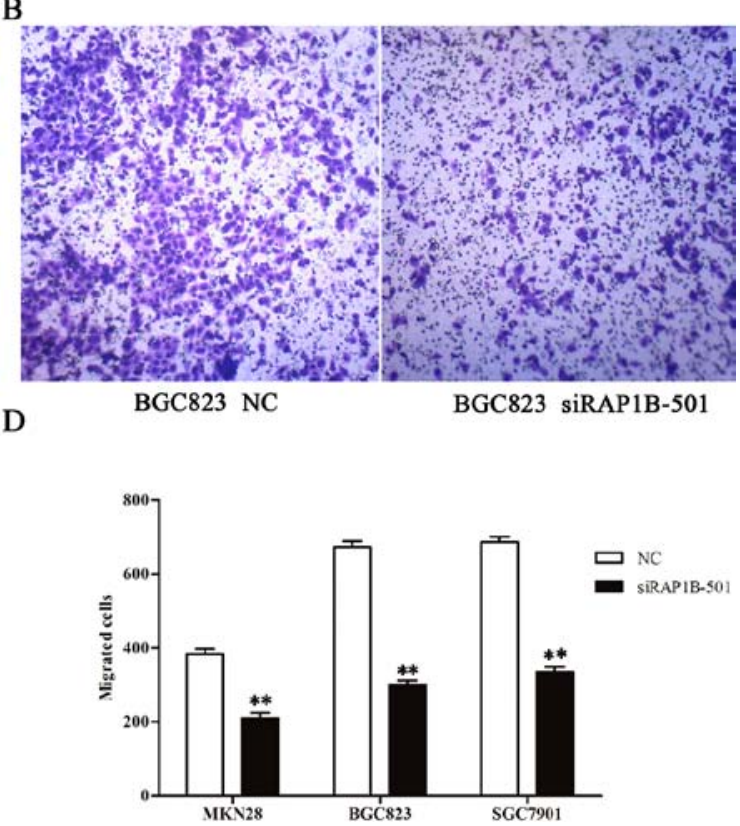

Figure 6. Effects of RAP1B knockdown on regulation of the invasion capacity of gastric cancer (GC) cells under a hypoxic condition. The invasion capacity was suppressed in GC cells transfected with siRNA RAP1B-501 under hypoxic conditions. (A) MKN28, (B) BGC823 and (C) SGC7901 cells; (D) the graph shows the quantified data of the assay $\left({ }^{* *} \mathrm{P}<0.001\right)$.

A
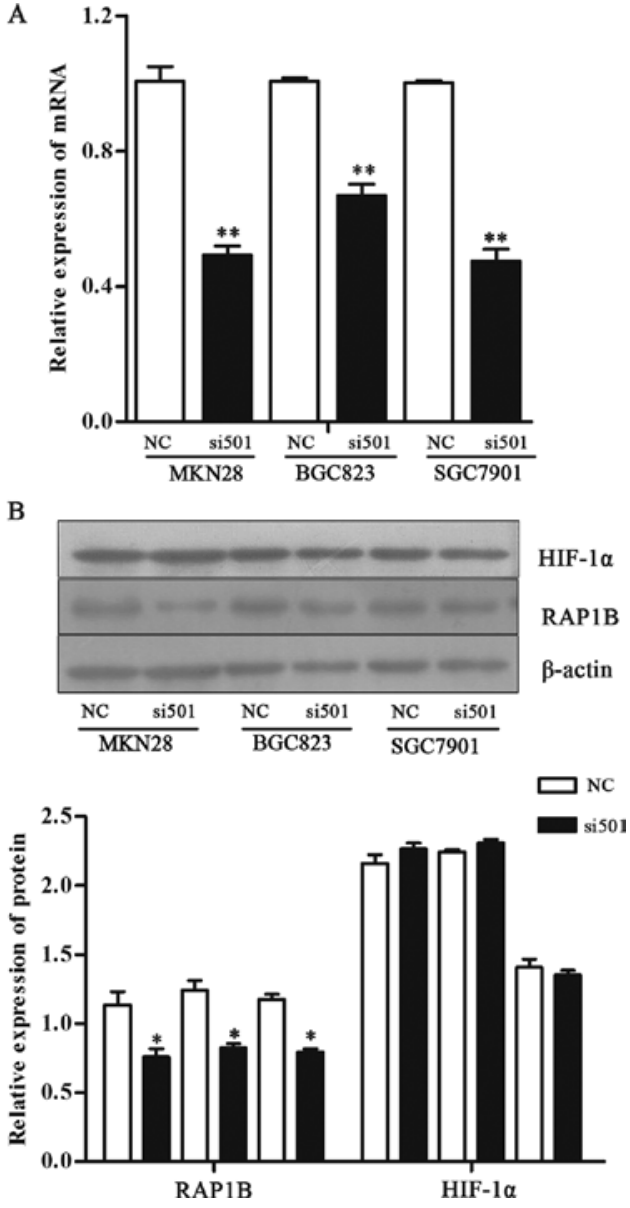

Figure 7. Expression of RAP1B and HIF-1 $\alpha$ in gastric cancer (GC) cells after transfection with siRNAs and cultured under a hypoxic condition. (A) qRT-PCR. Expression of RAP1B mRNA in GC cells after transfection with RAP1B siRNA-501 and treatment with $\mathrm{CoCl}_{2}$ and then subjected to qRT-PCR analysis ( $\left.{ }^{* *} \mathrm{P}<0.001\right)$, and $(\mathrm{B})$ western blotting. Duplicate cells from A were subjected to western blot analysis of RAP1B and HIF-1 $\alpha$ expression The graph shows the quantified data of the western blot analyses ( $\mathrm{P}<0.05)$.
Furthermore, hypoxia is a common feature of the tumor microenvironment, and the HIF-1 $\alpha$ level determines HIF-1 transcriptional activity under hypoxic conditions (28); thus, hypoxia and HIF- $1 \alpha$ regulate tumor cell migration and invasion through different gene pathways. For example, hypoxia and HIF- $1 \alpha$ are responsible for the migration and invasion of pancreatic ductal adenocarcinoma cells by elevating fascin overexpression (29). In addition, a previous study (30) demonstrated that under hypoxic conditions, the CD147 promoter is directly bound to HIF-1 $\alpha$ and that CD147 facilitates matrix metalloproteinase-2 activation in melanoma cells. Our current data showed that RAP1B expression in the cell nuclei was consistent with HIF-1 $\alpha$ expression in $\mathrm{GC}$ tissues. There is one similar study reporting that K-Ras causes high F-FDG accumulation and that HIF-1 $\alpha$ expression in addition increases F-FDG accumulation in hypoxic lesions (31). In addition, this is the first study to show that high RAP1B expression was detected under hypoxic conditions and that knockdown of RAP1B expression did not affect HIF-1 $\alpha$ expression, indicating that hypoxia and HIF-1 $\alpha$ could be upstream of RAP1B. However, it remains unknown how hypoxia and HIF-1 $\alpha$ upregulate RAP1B expression in GC cells.

RAP1B could be influenced and regulated by different factors. RAP1B is one isoform of RAP1, which is a member of the Ras superfamily of GTPases. The GTPases are molecular switches that cycle between an inactive GDP-bound state and an active GTP-bound state. The activation loop of GTPases is regulated by three different classes of proteins: guanylate exchange factors, guanylate activating proteins and guanylate dissociation inhibitors (32). Furthermore, RAP1B is also targeted by different mRNAs at the post-transcriptional level, regulating multiple cellular processes including proliferation, migration, invasion, metastasis and apoptosis (7,11,13-18). 
RAP1B is indirectly and directly impacted by different factors in various human cancers, implying that RAP1B serves as a therapeutic target in GC.

In conclusion, the present study showed a significant association of RAP1B expression with tumor TNM stage and regional lymph node metastasis. Hypoxia promoted the invasion capacity of GC cells by inducing the expression of RAP1B mRNA and protein. Our data indicate that RAP1B could be a useful prognostic marker of gastric carcinoma and may also serve as a therapeutic target.

\section{Acknowledgements}

The authors would like to thank Chen Huang and Xiao-Ge Zhao of the Department of Genetics and Molecular Biology, Medical School, Xi'an Jiaotong University for their technical support. The present study was supported by a grant from the National Natural Science Foundation of China (no. 81471710).

\section{References}

1. Ferro A, Peleteiro B, Malvezzi M, Bosetti C, Bertuccio P, Levi F, Negri E, La Vecchia $\mathrm{C}$ and Lunet $\mathrm{N}$ : Worldwide trends in gastric cancer mortality (1980-2011), with predictions to 2015, and incidence by subtype. Eur J Cancer 50: 1330-1344, 2014.

2. Jemal A, Bray F, Center MM, Ferlay J, Ward E and Forman D: Global cancer statistics. CA Cancer J Clin 61: 69-90, 2011.

3. Cervantes A, Roda D, Tarazona N, Roselló S and PérezFidalgo JA: Current questions for the treatment of advanced gastric cancer. Cancer Treat Rev 39: 60-67, 2013.

4. Ferlay J, Shin HR, Bray F, Forman D, Mathers C and Parkin DM: Estimates of worldwide burden of cancer in 2008: GLOBOCAN 2008. Int J Cancer 127: 2893-2917, 2010.

5. Ferlay J, Steliarova-Foucher E, Lortet-Tieulent J, Rosso S, Coebergh JW, Comber H, Forman D and Bray F: Cancer incidence and mortality patterns in Europe: Estimates for 40 countries in 2012. Eur J Cancer 49: 1374-1403, 2013.

6. Nakajima $\mathrm{H}$ and Kunimoto H: TET2 as an epigenetic master regulator for normal and malignant hematopoiesis. Cancer Sci 105: 1093-1099, 2014

7. Sheng Y, Ding S, Chen K, Chen J, Wang S, Zou C, Zhang J, Cao Y, Huang A and Tang H: Functional analysis of miR-101-3p and Raplb involved in hepatitis B virus-related hepatocellular carcinoma pathogenesis. Biochem Cell Biol 92: 152-162, 2014.

8. Lakshmikanthan S, Sobczak M, Chun C, Henschel A, Dargatz J, Ramchandran R and Chrzanowska-Wodnicka M: Rap1 promotes VEGFR2 activation and angiogenesis by a mechanism involving integrin $\alpha_{v} \beta_{3}$. Blood 118: 2015-2026, 2011.

9. Li Y, Yan J, De P, Chang HC, Yamauchi A, Christopherson KW II, Paranavitana NC, Peng X, Kim C, Munugalavadla V, et al: Rapla null mice have altered myeloid cell functions suggesting distinct roles for the closely related Rapla and $1 \mathrm{~b}$ proteins. J Immunol 179: 8322-8331, 2007.

10. Wittchen ES, Aghajanian A and Burridge K: Isoform-specific differences between Rap1A and Rap1B GTPases in the formation of endothelial cell junctions. Small GTPases 2: 65-76, 2011.

11. Lin KT, Yeh YM, Chuang CM, Yang SY, Chang JW, Sun SP, Wang YS, Chao KC and Wang LH: Glucocorticoids mediate induction of microRNA-708 to suppress ovarian cancer metastasis through targeting Rap1B. Nat Commun 6: 5917, 2015.

12. Kumar S, Xu J, Kumar RS, Lakshmikanthan S, Kapur R, Kofron M, Chrzanowska-Wodnicka M and Filippi MD: The small GTPase Raplb negatively regulates neutrophil chemotaxis and transcellular diapedesis by inhibiting Akt activation. J Exp Med 211: 1741-1758, 2014.

13. Zhang M, Zhou S, Zhang L, Zhang J, Cai H, Zhu J, Huang C and Wang J: miR-518b is down-regulated, and involved in cell proliferation and invasion by targeting Raplb in esophageal squamous cell carcinoma. FEBS Lett 586: 3508-3521, 2012.
14. Bischoff A, Huck B, Keller B, Strotbek M, Schmid S, Boerries M, Busch H, Müller D and Olayioye MA: miR149 functions as a tumor suppressor by controlling breast epithelial cell migration and invasion. Cancer Res 74: 5256-5265, 2014.

15. Shen K, Mao R, Ma L, Li Y, Qiu Y, Cui D, Le V, Yin P, Ni L and Liu J: Post-transcriptional regulation of the tumor suppressor miR-139-5p and a network of miR-139-5p-mediated mRNA interactions in colorectal cancer. FEBS J 281: 3609-3624, 2014.

16. Peng H, Luo J, Hao H, Hu J, Xie SK, Ren D and Rao B: MicroRNA-100 regulates SW620 colorectal cancer cell proliferation and invasion by targeting RAP1B. Oncol Rep 31: 2055-2062, 2014.

17. She X, Yu Z, Cui Y, Lei Q, Wang Z, Xu G, Luo Z, Li G and Wu M: miR-181 subunits enhance the chemosensitivity of temozolomide by Rap1B-mediated cytoskeleton remodeling in glioblastoma cells. Med Oncol 31: 892, 2014.

18. Guo H, Hu X, Ge S, Qian G and Zhang J: Regulation of RAP1B by miR-139 suppresses human colorectal carcinoma cell proliferation. Int J Biochem Cell Biol 44: 1465-1472, 2012.

19. Malchinkhuu E, Sato K, Maehama T, Ishiuchi S, Yoshimoto Y, Mogi C, Kimura T, Kurose H, Tomura H and Okajima F: Role of Rap1B and tumor suppressor PTEN in the negative regulation of lysophosphatidic acid - induced migration by isoproterenol in glioma cells. Mol Biol Cell 20: 5156-5165, 2009.

20. Ruan K, Song G and Ouyang G: Role of hypoxia in the hallmarks of human cancer. J Cell Biochem 107: 1053-1062, 2009.

21. Vaupel P and Mayer A: Hypoxia in cancer: Significance and impact on clinical outcome. Cancer Metastasis Rev 26: 225-239, 2007.

22. Wong CC, Kai AK and Ng IO: The impact of hypoxia in hepatocellular carcinoma metastasis. Front Med 8: 33-41, 2014.

23. Chen L, Shi Y, Yuan J, Han Y, Qin R, Wu Q, Jia B, Wei B, Wei L, Dai G, et al: HIF-1 alpha overexpression correlates with poor overall survival and disease-free survival in gastric cancer patients post-gastrectomy. PLoS One e90678: 2014.

24. Semenza GL: Targeting HIF-1 for cancer therapy. Nat Rev Cancer 3: 721-732, 2003.

25. Edge S, Byrd DR, Compton CC, Fritz AG, Greene FL and Trotti A (eds): AJCC Cancer Staging Handbook, Springer, New York, 2010.

26. Mitra RS, Zhang Z, Henson BS, Kurnit DM, Carey TE and D'Silva NJ: Rap1A and rap1B ras-family proteins are prominently expressed in the nucleus of squamous carcinomas: Nuclear translocation of GTP-bound active form. Oncogene 22: 6243-6256, 2003.

27. Ntantie E, Gonyo P, Lorimer EL, Hauser AD, Schuld N, McAllister D, Kalyanaraman B, Dwinell MB, Auchampach JA and Williams CL: An adenosine-mediated signaling pathway suppresses prenylation of the GTPase Rap1B and promotes cell scattering. Sci Signal 6: ra39, 2013.

28. Bruick RK: Oxygen sensing in the hypoxic response pathway: Regulation of the hypoxia-inducible transcription factor. Genes Dev 17: 2614-2623, 2003.

29. Zhao X, Gao S, Ren H, Sun W, Zhang H, Sun J, Yang S and Hao J: Hypoxia-inducible factor-1 promotes pancreatic ductal adenocarcinoma invasion and metastasis by activating transcription of the actin-bundling protein fascin. Cancer Res 74 : 2455-2464, 2014.

30. Zeng W, Su J, Wu L, Yang D, Long T, Li D, Kuang Y, Li J, Qi M, Zhang J, et al: CD147 promotes melanoma progression through hypoxia-induced MMP2 activation. Curr Mol Med 14: 163-173, 2014.

31. Iwamoto $\mathrm{M}$, Kawada $\mathrm{K}$, Nakamoto $\mathrm{Y}$, Itatani $\mathrm{Y}$, Inamoto $\mathrm{S}$, Toda K, Kimura H, Sasazuki T, Shirasawa S, Okuyama H, et al: Regulation of ${ }^{18} \mathrm{~F}$-FDG accumulation in colorectal cancer cells with mutated KRAS. J Nucl Med 55: 2038-2044, 2014.

32. Citalán-Madrid AF, García-Ponce A, Vargas-Robles H, Betanzos A and Schnoor M: Small GTPases of the Ras superfamily regulate intestinal epithelial homeostasis and barrier function via common and unique mechanisms. Tissue Barriers 1: e26938, 2013 